\title{
Bio-Inspired Non-Cooperative Multi-Robot Herding
}

\author{
Alyssa Pierson $^{1}$ and Mac Schwager ${ }^{2}$
}

\begin{abstract}
This paper presents a new control strategy to control a group of dog-like robots to drive a herd of noncooperative sheep-like agents to a goal region in the environment. The sheep-like agents, which may be biological or robotic, respond to the presence of the dog-like robots with a repelling potential field common in biological models of the behavior of herding animals. Our key insight in designing control laws for the dog-like robots is to enforce geometrical relationships that allow for the combined dynamics of the dogs and sheep to be mapped to a simple unicycle robot model. We prove convergence of a single sheep to a desired goal region using two or more dogs, and we propose a control strategy for the case of any number of sheep driven by two or more dogs. Simulations in Matlab and hardware experiments with Pololu m3pi robots demonstrate the effectiveness of our control strategy.
\end{abstract}

\section{INTRODUCTION}

We consider the problem of non-cooperative herding, analogous to dogs coordinating their motions to drive a herd of sheep to a goal location. In this system, the "sheep" agents naturally run away from the "dog" robots, and by designing controllers for the dogs, we can drive the sheep to some desired region in the environment. We propose a feedback control strategy for the dogs to coordinate their positions with one another to so as to partially encircle the herd. The dogs use this partial encirclement to apply pressure to the herd to move it in a desired direction, and can thereby steer the herd towards the goal. First, we introduce control strategies for two dogs controlling a single sheep, and show that under certain geometrical constraints, the dynamics of this system can be reduced to the well-known unicycle kinematic robot. We use this insight to map a simple linear control strategy for the unicycle robot back into a nonlinear feedback control law for the two dogs. We generalize this approach to the case of an arbitrary number of dogs driving a single sheep, and finally to the general case of multiple dogs driving multiple sheep. We demonstrate the performance of the control strategies in Matlab simulations, and in hardware experiments with Pololu $\mathrm{m} 3$ pi robots in a motion capture environment.

Although we will use the dog-sheep analogy throughout this paper, it is understood that the "dogs" are robotic agent under our control, while the sheep may be biological herding animals, or other robots that behave like herding animals. The herding agents respond to the dogs with a repulsive

This work was supported in part by NSF grants CNS-1330036 and IIS1350904.

${ }^{1}$ Alyssa Pierson is with the Department of Mechanical Engineering, Boston University, Boston, MA 02215 pierson@bu.edu

${ }^{2}$ Mac Schwager is with the Department of Mechanical Engineering and the Division of Systems Engineering, Boston University, Boston, MA 02215 schwager@bu.edu potential field commonly used to model the response of herding animals to perceived threats. Our control strategy could be useful in wildlife management, as well as other applications. For example, in Australia, helicopters are used to muster cattle for large-scale relocation. This dangerous profession requires pilots to fly at low altitudes and perform quick maneuvers, which results in as many as 10 deaths per year [1]. The lives of these human pilots could be saved if our control strategy was implemented on teams of UAVs to autonomously muster cattle. Another application is managing wildlife populations in national parks, where it is necessary to monitor animals, as well as steer them away from environmental dangers. Our controllers may also apply to the problem of micro-manipulation of bacteria with magnetic fields [2]. Human crowds may also be controlled and directed by robots in an emergency evacuation using our control strategy. The controller we describe may also offer a plausible biological hypothesis for how real dogs herd sheep.

We consider this as a non-cooperative multi-robot problem, since the objective of the dogs is to steer the sheep, while the sheep are not actively inclined nor opposed to being steered. This scenario lies somewhere between a fully a cooperative setting, in which all robots work towards the goal, and a fully adversarial setting, in which two teams of robots work against each other toward opposite goals.

\section{A. Related Work}

There has been surprisingly limited prior work on noncooperative robotic herding. One exception is Vaughan's pioneering work [3], [4], in which a single robot is used to herd ducks in a specially design experiment arena. More recently, Lien et. al developed a set of behavior primitives for controlling a flock with multiple shepherds [5]. In contrast to both of these, our work takes a control theoretic approach to design feedback laws for an arbitrary number of dogs to drive an arbitrary number of sheep. Other authors have formulated the problem as a dynamic pursuit-evasion game to find optimal trajectories that allow the herder to drive the sheep to some goal position [6], [7]. In this work, the herder "catches" the sheep at the goal location, whereas in our setting the herders relocate the herd without the intent to "catch" it. Furthermore, in the area of multi-agent formation control, researchers have considered driving robots into a desired formation [8], [9]. In this setting, the robots typically have linear dynamics, and have cooperative control laws that are intended to move them into a formation, while in contrast, our herd of sheep are non-cooperative, and have a nonlinear response to the dogs.

To model the herd dynamics, we use potential fields, which 
is common in animal aggregation modeling for schools of fish [10], birds, slime molds, mammal herds, and other swarms [11], [12]. These models have been applied to multi-agent systems to simulate flocking [13], cooperative group control [14], [15], [16], and interaction with collision avoidance [17].

Our work proposes a reduction from the nonlinear dogsheep system to the well-known unicycle model for a differential drive robot [18], [19]. This introduces a nonholonomic constraint, which limits the robot to only translate in the direction of its heading. Several techniques to drive the unicycle robot to the origin without violating Brockett's Theorem [20] include optimal control [21], [22], sliding mode control [23], or Lyapunov-like functions [24]. Our chosen strategy is to control a point that is offset from the center of mass of the robot, whose dynamics then become holonomic [25]. We call this control strategy a point-offset controller. By designing feedback controllers for the point offset, we obtain nonlinear feedback controllers for the dogs, which in turn drive the sheep to a goal region in the environment.

The remainder of the paper is organized as follows. In Section II we present our mathematical formulation of the problem. Section III builds the kinematic models for the various numbers of dogs and sheep and describes the reduction to a unicycle robot. We propose a two part control strategy in Section IV. Results of simulation and experiments are given in Sections V and VI, respectively, and we give our conclusions in Section VII.

\section{Problem Formulation}

Consider $m$ herders (or "dogs") with positions $d_{j} \in \mathbb{R}^{2}$, where $j \in\{1, \ldots, m\}$ and $n$ herd members (or "sheep") with positions $s_{i} \in \mathbb{R}^{2}$, where $i \in\{1, \ldots, n\}$. The "dogs" in this model are presumed to be robots since they are under our control, while the herd members can be robots, sheep, cattle, other herding animals, or even humans. However, for the purposes of this paper we will use the shepherding analogy throughout. We will assume the dogs have integrator dynamics,

$$
\dot{d}_{j}=u_{j}
$$

Here, $u_{j}$ is the control input moving $d_{j}$ through the environment. Our main goal is to design $u_{j}$ such that the dogs drive the sheep to some goal region. We will model the sheep's repulsion from the dogs using an artificial potential field [14], which is common in robotics and in models of biological herding animals. Using the potential field $W=$ $\sum_{i=1}^{n} \sum_{j=1}^{m} \frac{1}{\left\|d_{j}-s_{i}\right\|}$, we obtain

$$
\dot{s}_{i}=\frac{\partial W}{\partial s_{i}}=\sum_{j=1}^{m} \frac{-\left(d_{j}-s_{i}\right)}{\left\|d_{j}-s_{i}\right\|^{3}} .
$$

For now we do not consider the additional forces from flocking dynamics between members of the herd, although this will be introduced later in Section III.

Also consider a user-defined goal region

$$
B_{\ell}(g)=\left\{q \in \mathbb{R}^{2} \mid\|q-g\| \leq \ell\right\}
$$

centered at a goal point $g \in \mathbb{R}^{2}$ with a desired radius $\ell>0$. This goal region represents the set of allowable final configurations for the sheep to occupy. Without loss of generality, we can define our coordinate frame to be centered at the goal point, so that $g=0$. We take the goal point to be the origin through the rest of the paper.

Problem 1: (Multi-Agent Herding) Given the dynamics of the herd (2), find control laws $u_{j}=f(d, s)$ for $d_{j}$ herders with dynamics (1) to relocate the herd from arbitrary initial conditions to the desired region in the environment $B_{\ell}(g)$.

\section{Kinematic Modeling And Reduction to UNICYCLE}

We propose a solution to Problem 1 that is both simple and scalable to $m$ herders. The key insight of our approach lies in enforcing geometrical relationships that map the complex, nonlinear dog and sheep dynamics to a simple unicycle model. This creates an ideal unicycle-like system which we will utilize in our controller design. We will first introduce terminology and basic nomenclature to describe the unicycle model, then present our herding models that reduce to the unicycle-like system.

\section{A. Ideal Unicycle Model}

Consider the nonholonomic vehicle model shown in Figure 1. For a nonholonomic vehicle, we can define a local reference frame $Q$ relative to the global base frame $B$. Its forward velocity $v$ defines the local $q_{x}$ direction, as shown on the right in Figure 1. The orientation $\phi$ relates the heading $q_{x}$ to the global $b_{x}$, and the angular velocity is defined as $w=\dot{\phi}$.

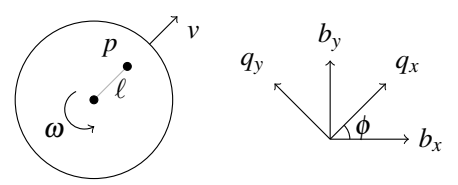

Fig. 1. Nonholonomic vehicle model

Consider also some point offset $p$ a distance $\ell$ from the center of the vehicle. While the unicycle-like vehicle has nonholonomic dynamics, it turns out that $p$ is holonomic. It can be shown that the dynamics of $\dot{p}$ can be related to $v$ and $w$ as [25]

$$
\left[\begin{array}{l}
v \\
\omega
\end{array}\right]=\left[\begin{array}{cc}
\cos (\phi) & \sin (\phi) \\
-\frac{\sin (\phi)}{\ell} & \frac{\cos (\phi)}{\ell}
\end{array}\right] \dot{p} .
$$

In Section IV, we will introduce our control strategy for the point offset $p$, which relates to the unicycle using (3).

\section{B. Single-Sheep Model}

Instead of allowing the dogs to occupy any point in the environment, consider the case where all dogs are a fixed distance $r$ from the sheep. We can show that when this occurs, the system dynamics reduce to a unicycle-like vehicle. First, we introduce basic concepts in our kinematic model with a single sheep and two dogs, then we generalize this to any $m$ dogs. 
1) Single Sheep with Two Dogs: We begin with the case of $n=1$ sheep and $m=2$ dogs, shown in Figure 2 .

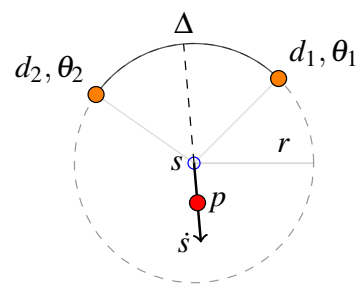

Fig. 2. Configuration of two dogs and a single sheep

Figure 2 illustrates the configuration where both dogs are located some distance $r$ from the sheep. The position of the dogs $d_{j}$ can then be written in terms of their angular orientation $\theta_{j}$ relative to the sheep as

$$
d_{j}=s+r\left[\begin{array}{c}
\cos \left(\theta_{j}\right) \\
\sin \left(\theta_{j}\right)
\end{array}\right] .
$$

Furthermore, the dynamics of the herd introduced in (2) can be simplified as

$$
\dot{s}=\frac{-1}{r^{2}}\left[\begin{array}{l}
\sum \cos \left(\theta_{j}\right) \\
\sum \sin \left(\theta_{j}\right)
\end{array}\right] .
$$

To maintain this kinematic relationship, the dynamics of the dogs in (1) must take the form

$$
\dot{d}_{j}=\dot{s}+r \dot{\theta}_{j}\left[\begin{array}{c}
-\sin \left(\theta_{j}\right) \\
\cos \left(\theta_{j}\right)
\end{array}\right] \text {. }
$$

Similar to our unicycle model, we can define the heading $\phi$ as the direction of $\dot{s}$ relative to the base frame, where

$$
\phi=\frac{1}{2}\left(\theta_{1}+\theta_{2}\right)+\pi \text {. }
$$

We can also see that $d_{1}$ and $d_{2}$ are always symmetric around the line formed by $\dot{s}$. Consider the angular separation between the two herders as $\Delta=\theta_{2}-\theta_{1}$. Thus, we can rewrite the angles in terms of $\phi$ and $\Delta$ as

$$
\theta_{1}=\phi+\pi-\frac{\Delta}{2}, \quad \theta_{2}=\phi+\pi+\frac{\Delta}{2} .
$$

These simplifications of the angle in (7) will allow us to distill the complex dynamics of the herders into two main state variables, $\phi$ and $\Delta$, which makes it much simpler to describe the dynamics when considering $m$ dogs.

2) Single Sheep with $m$ Dogs: To generalize to $m$ dogs, we will assume equal spacing of the dogs between $d_{1}$ and $d_{m}$ along the desired radius, as shown in Figure 3. Thus, $\Delta$ becomes the total separation between the first $\operatorname{dog} d_{1}$ and last $\operatorname{dog} d_{m}$.

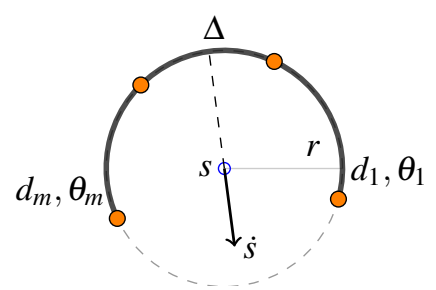

Fig. 3. Configuration of $m$ dogs and one sheep.
The angular orientation of each dog with respect to $\phi$ can be expressed as

$$
\theta_{j}=\phi+\pi+\Delta_{j}
$$

where

$$
\Delta_{j}=\Delta \frac{(2 j-m-1)}{(2 m-2)} .
$$

Substituting (8) into (5), the sheep dynamics become

$$
\dot{s}=\frac{-1}{r^{2}}\left[\begin{array}{c}
\sum \cos \left(\theta_{j}\right) \\
\sum \sin \left(\theta_{j}\right)
\end{array}\right]=\frac{-\sin \left(\frac{m \Delta}{2-2 m}\right)}{r^{2} \sin \left(\frac{\Delta}{2-2 m}\right)}\left[\begin{array}{c}
\cos (\phi) \\
\sin (\phi)
\end{array}\right]
$$

which allows us to describe the dynamics of the sheep using only the two state variables, $\phi$ and $\Delta$, despite having $m$ dogs. Similarly, by substituting (8) in (6), the dynamics for the dogs become

$$
\dot{d}_{j}=\dot{s}+r\left(\dot{\phi}+\dot{\Delta}_{j}\right)\left[\begin{array}{c}
\sin \left(\phi+\Delta_{j}\right) \\
-\cos \left(\phi+\Delta_{j}\right)
\end{array}\right] .
$$

By defining the orientation of the dogs in terms of $\phi$ and $\Delta$ along some radius in (8) and restricting the dogs' kinematics to obey (10), we can map these quantities to the angular and linear velocity of a unicycle-like vehicle.

Remark 1: Note that this model assumes the dogs are fixed on some circle of radius $r$ relative to the herd, which limits the initial configurations of the dogs relative to the sheep. Later, we will introduce a tracking controller for the dogs that allows them to start anywhere in the environment and converge upon this configuration. We also present a radial controller in (14) to adjust the radius used by the dogs online when controlling multiple sheep.

Proposition 1: The herding dynamics in (6) and (5) can be reduced to an equivalent unicycle model with forward velocity $v$ and orientation $\phi$.

Proof: To see this mapping, note that the direction of the unicycle's velocity $v$ and the direction of the herd's velocity $\dot{s}$ are both $\phi$. As for the velocity, we can find

$$
v=\|\dot{s}\|=\frac{\sin \left(\frac{m \Delta}{2-2 m}\right)}{r^{2} \sin \left(\frac{\Delta}{2-2 m}\right)} .
$$

Note that for (11), there are an infinite number of possible values of $\Delta$ for a given value of $v$. However, over the range of $\Delta=(0,2 \pi)$, this mapping is one-to-one. Thus, for a given velocity, we can find the corresponding $\Delta$.

The remaining quantities in the mapping are $\dot{\phi}$ and $\dot{\Delta}$ in the herder's dynamics (10). We can directly map $\dot{\phi}=\omega$ from the unicycle dynamics. The dynamics for $\dot{\Delta}$ can also be found from the dynamics of $\dot{v}$.

Ultimately, we will use Proposition 1 in our controller design of the system. Instead of trying to determine individual controllers for all of the dogs, we will instead design controllers for the ideal unicycle-like system. Based on the idealized system, we can find controllers for the dogs that will enforce this behavior. 


\section{Multi-Sheep Model}

Now, consider the case of $m$ dogs and $n$ sheep. In the case of multiple sheep, we define the radius $r$ from the mean of the herd, $\bar{s}$, as shown in Figure 4.

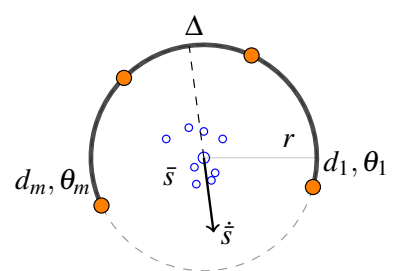

Fig. 4. Configuration of $m$ dogs and $n$ sheep.

Here, the dynamics of the herd mean are

$$
\dot{\bar{s}}=\frac{1}{n} \sum_{i=1}^{n} \sum_{j=1}^{m} \frac{-\left(d_{j}-s_{i}\right)}{\left\|d_{j}-s_{i}\right\|^{3}} .
$$

Due to the varying nature of the extent of the herd, we also introduce an additional term $\dot{r}$ in the controller to regulate the radius $r$. The dynamics are then written

$$
\dot{d}_{j}=\dot{\bar{s}}+r \dot{\theta}_{j}\left[\begin{array}{c}
-\sin \left(\theta_{j}\right) \\
\cos \left(\theta_{j}\right)
\end{array}\right]+\dot{r}\left[\begin{array}{c}
\cos \left(\theta_{j}\right) \\
\sin \left(\theta_{j}\right)
\end{array}\right] .
$$

We design the radius controller $\dot{r}$ to maintain some desired radius $r_{0}$, as well as adjust for the standard deviation of the herd. Our proposed controller is

$$
\dot{r}=\left(r_{0}-r\right)+\frac{1}{n} \sum_{i=1}^{n} 2\left(s_{i}-\bar{s}\right)^{T}\left(\dot{s}_{i}-\dot{\bar{s}}\right) .
$$

where $r_{0}$ is the desired radius if the herd were a single sheep.

\section{Controller Design}

Section III introduced geometric constraints on the system, which allow us to map the kinematics of the herding system to a unicycle-like vehicle. Our goal, as stated in Problem 1 , is to drive the herd to some ball around the origin. To control this system, in this section we propose a controller that drives a point-offset of the unicycle-like system to the origin. Given some desired velocity that controls the ideal system towards the goal region, we can calculate desired positions for the dogs along the circumference of the circle. We then employ a tracking controller to drive the dogs to these desired positions. For our analysis, we assume the dynamics of the ideal unicycle-like system are significantly slower than the dynamics to drive the dogs to their ideal positions.

To design the tracking controller, consider the desired position, $d_{j}^{*}$, for each dog. This ideal position occurs when the dogs lie on a circle of radius $r$ around the sheep, with spacing $\Delta_{j}$. Let the ideal orientation, $\phi^{*}$, be the angle that points the herd's velocity towards the origin. To find the ideal velocity for the unicycle-like system, we will find a controller for a point-offset $p$ from the sheep that drives the point offset to the origin. While there exist many possible choices for controlling the point-offset $p$, we opt for a simple proportional feedback controller,

$$
\dot{p}=-k p \text {. }
$$

Plugging this into our mapping in (3), we find the ideal velocity becomes

$$
v^{*}=\left[\begin{array}{ll}
\cos (\phi) & \sin (\phi)
\end{array}\right](-k p) .
$$

Using the mapping in (11), we can then determine the desired separation $\Delta_{j}^{*}$ for the dogs. Overall, this yields the desired position of the dogs, written as

$$
d_{j}^{*}=s+r\left[\begin{array}{c}
\cos \left(\phi^{*}+\Delta_{j}^{*}\right) \\
-\sin \left(\phi^{*}+\Delta_{j}^{*}\right)
\end{array}\right] .
$$

Finally, our tracking controller becomes

$$
\dot{d}_{j}=-K_{d}\left(d_{j}^{*}-d_{j}\right)
$$

Under the following mild assumption, we can analyze the performance of this control strategy.

Assumption 1: The desired dog positions $d_{j}^{*}(16)$ evolve slowly enough compared to the speed of our dogs $\dot{d}_{j}$ (17) that we can approximate them as being fixed.

Under Assumption 1, the desired positions $d_{j}^{*}$ are constant and the tracking controller $(17), d_{j}$ converges exponentially to $d_{j}^{*}$. In practice, this allows us to the start the dogs from any point within the environment, and they will converge upon the ideal unicycle-like system. The following algorithm summarizes the steps in the controller. Note that when there are multiple sheep in the herd, the radius is continuously updated using our radial controller (14). In the case of a single sheep, the radius is always constant.

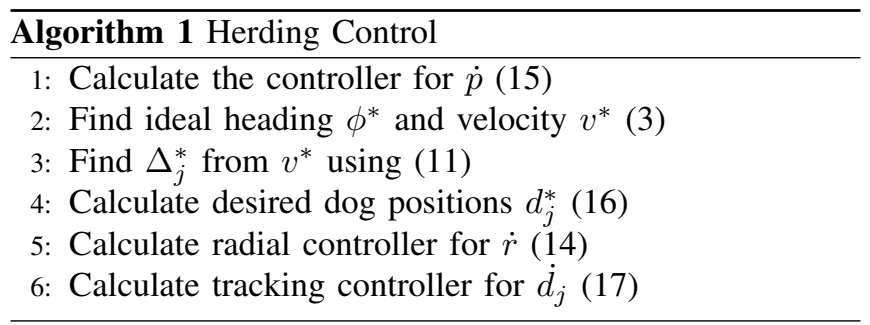

Using our assumption that the tracking controller allows the dogs to converge upon their ideal positions, we can analyze the system as if it were the simple unicycle-like system. Before presenting our main proposition, we will first define some necessary quantities. The point-offset is defined relative to the herd in the direction of the herd's velocity. Let $Q$ be the local coordinate frame of the herd, with $q_{x}$ defined in the direction of the herd's velocity, shown in Figure 1, and written as

$$
q_{x}=\left[\begin{array}{c}
\cos (\phi) \\
\sin (\phi)
\end{array}\right] \quad q_{y} \perp q_{x} .
$$

Our point offset $p$ and its derivative can then be written

$$
\begin{aligned}
& p=s+\ell q_{x} \\
& \dot{p}=\dot{s}+\ell \dot{\phi} q_{y},
\end{aligned}
$$


We can also write $s$ and $\dot{s}$ in the $Q$ frame as

$$
s=s_{x} q_{x}+s_{y} q_{y}, \quad \dot{s}=v q_{x}
$$

where $v$ is the norm of the herd's velocity in the global reference frame. Combined, the expressions for $p$ and $\dot{p}$ become

$$
\begin{aligned}
p & =\left(s_{x}+\ell\right) q_{x}+s_{y} q_{y}, \\
\dot{p} & =v q_{x}+\ell \dot{\phi} q_{y} .
\end{aligned}
$$

Recall that we chose a simple feedback control for our pointoffset in (15). Using our mapping in (3), we can write the velocity and angular velocity as

$$
v=-k q_{x}^{T} p, \quad w=\dot{\phi}=\frac{-k}{\ell} q_{y}^{T} p .
$$

We are now ready to state our main proposition on the behavior of a single sheep and $m$ dogs.

Proposition 2: For the single sheep, $m$ dog system described in (9) and (10), with the tracking controller in (17), the herd converges to the ball of radius $l$ about the origin, $B_{\ell}$.

Proof: For our unicycle-like system centered around the sheep, the point offset $p$ is defined in (19). It is equivalent to say that if the point offset converges to the origin, the herd converges to the ball $B_{\ell}$ about the origin. Consider the Lyapunov candidate function

$$
V=\frac{1}{2} p^{T} p
$$

with derivative

$$
\dot{V}=p^{T} \dot{p}
$$

Substituting our expression for $\dot{p}(21)$, this becomes

$$
\dot{V}=p^{T}\left(v q_{x}+\ell \dot{\phi} q_{y}\right) \text {. }
$$

For our unicycle system, we can then plug in the expressions for $v$ and $\dot{\phi}$ chosen in (22), thus

$$
\begin{aligned}
\dot{V} & =p^{T}\left(-k\left(q_{x}^{T} p\right) q_{x}+-k\left(q_{y}^{T} p\right) q_{y}\right) \\
& =-k\|p\|^{2}<0 .
\end{aligned}
$$

From Lyapunov theory [26], the equilibrium point $p^{*}=0$ is asymptotically stable. Furthermore, when $p=0$, the sheep are at most a distance $\ell$ away from the origin, thus proving Proposition 2.

\section{Simulations}

The following simulations were performed in Matlab to demonstrate the capabilities of our herding algorithm. First, we present simulations illustrating the case of $n=1$ sheep with $m$ dogs. Despite starting from random configurations, our system converges to the dynamics of the ideal unicyclelike vehicle, and we can successfully relocate the herd to a ball around the origin. We also demonstrate our algorithm for multiple sheep, and also investigate the effects of including additional inter-agent repelling and attracting forces among the sheep.

\section{A. Herding with $n=1$ Sheep}

Our first simulation shows the case of $m=4$ dogs and a single sheep. Figure 5 illustrates the configuration and trajectories of all agents over time. In the figure, the green $\mathrm{x}$ represents the goal point, and the green circle denotes the goal region $B_{\ell}(g)$. The blue squares denote the dogs, and black circle and $x$ are the sheep and point offset, respectively.

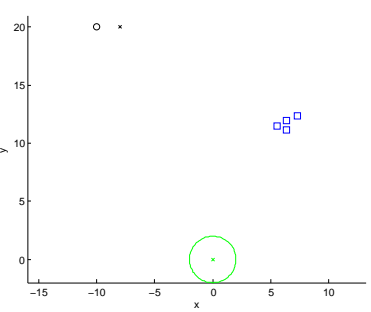

(a)

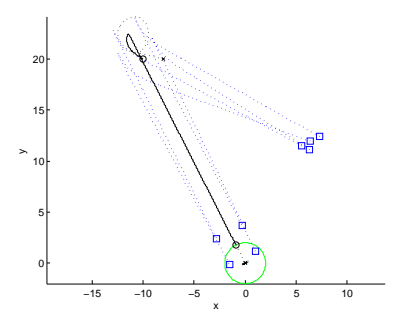

(b)
Fig. 5. (a) Initial configuration of $m=4$ herders and single sheep moving towards goal region. (b) Trajectory of the agents and the point offset, ending within the goal region.

We can see from Figure 5 that the dogs do not start near the sheep, but converge to a circle around the sheep, which then drives the point-offset to the origin. To illustrate the convergence over time, Figure 6 compares the distance between the point offset $(\|p\|)$ and the goal over 30 trials. The initial starting locations were randomized for each agent in each of the trials, yet we see in all simulations the point-offset converges to the origin, validating our claims in Proposition 2.

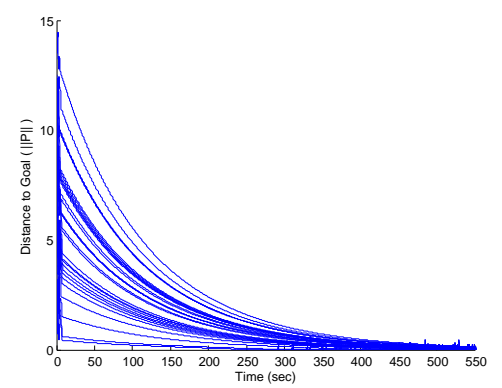

Fig. 6. Convergence of the point offset to the origin over 30 trials.

\section{B. Herding with $n>1$ Sheep}

For the case of multiple sheep with $m$ dogs, we add interagent forces between the sheep in the herd in addition to the repulsion forces the sheep experience from the dogs. For the purposes of these simulations, we use the flocking dynamics presented in Vaughan's work [3], [4] for inter-agent forces. Figure 7 shows two examples of controlling multiple sheep. The controllers use the herd mean $\bar{s}$, as well as the radial controller presented in (14). For the simulations presented in Figure 7, the inter-herd forces have low repulsion relative to the distances to the dogs, meaning the sheep act as a cohesive unit. With these properties, the dogs are still able 
to control the group to some goal region using our pointoffset controller on the mean of the herd.

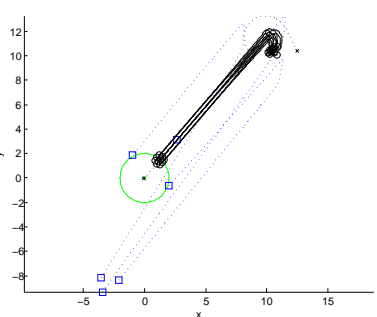

(a)

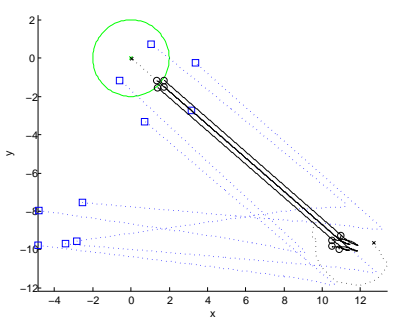

(b)
Fig. 7. (a) Trajectories of $m=3$ dogs and $n=10$ sheep moving towards goal region. (b) Trajectories of $m=5 \operatorname{dogs}$ and $n=4$ sheep moving towards goal region.

On the other hand, if we set the flocking dynamics to have higher repulsive forces between the herd members, the sheep have a greater tendency to disperse. Figure 8 shows the trajectories of two simulations where the sheep experience high repulsive forces.
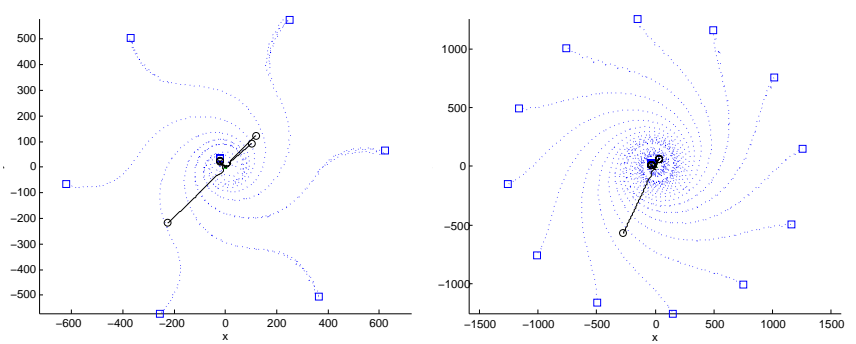

Fig. 8. Trajectories of $m=6$ dogs and single $n=3$ sheep [left] and $m=12$ dogs, $n=10$ sheep [right] with higher inter-herd repulsive forces relative to the distance to the dogs.

As seen in Figure 8, when the sheep have high repulsive forces between each other, the group spirals away from the mean. Surprisingly, the point offset from the mean still remains near the origin. Figure 9 compares the average distances of the sheep, dogs, and point offset for lowrepulsive and high-repulsive inter-agent forces among the sheep, illustrating that even though Figure 8 may look unsuccessful, the controller is still working as designed.
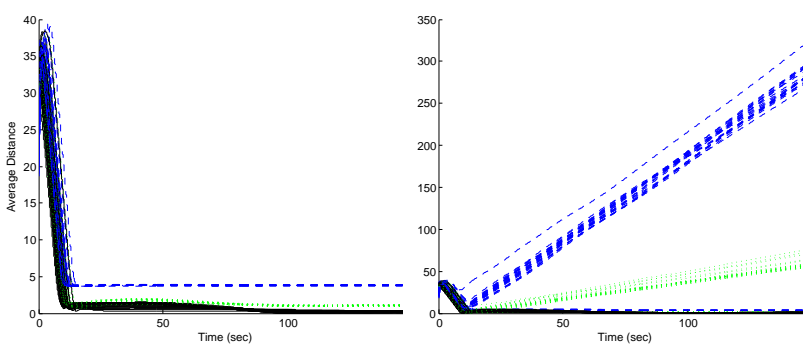

Fig. 9. Average distances from the origin for the point offset (black), herd members (green), and dogs (blue) over the 30 trials. The left figure has low repulsive forces, while the right uses high repulsive forces.

Note that the only metric in the radial controller is to adjust for the variance, but there is nothing in the current controller design to decrease the variance. We see from Figure 9 that in both cases, the point offset (black) is driven to the origin. As the sheep disperse under high repulsive forces, the dogs also disperse, but overall keep the mean of the herd near the origin. Future work will investigate modifications to the control strategy that gives guarantees on the final variance of the herd.

\section{EXPERIMENTS}

To demonstrate our algorithm, experiments were conducted at Boston University in the Multi-Robot Systems Lab. The lab utilizes an OptiTrack ${ }^{1}$ system with sixteen IR cameras to track reflective markers and provide real-time localization. We use Pololu's $\mathrm{m} 3 \mathrm{pi}^{2}$ robot equipped with an mbed microcontroller and $\mathrm{XBee}^{3}$ radio. Position data is obtained by MATLAB, which is then used to compute control laws and send information to the $\mathrm{m} 3$ pi robots via the XBee radio. Due to the limitations of the mbed microcontroller, computation is done on a central computer and only updated velocity information is sent to the $\mathrm{m} 3$ pi robots. Future experiments will explore moving the algorithm on board. Four short-throw projectors are used to display the goal point and simulated quantities on the floor mats during the experiment.

The biggest challenge during implementation was the culmination in system inefficiencies not present in simulation. Using two computers introduces time delays in the system, coupled with latencies caused by sending commands via Matlab over the XBee radio. While our simulations assume that all robots have holonomic dynamics, the $\mathrm{m} 3$ pis are nonholonomic vehicles with noisy, lossy actuation. In addition, the floor mats in the lab introduce a friction force on the robots, requiring them to travel at a minimum speed.

Combined, the experiments include unmodeled behaviors that are hard to predict or quantify in a simulation. Despite these challenges, we were still able to perform successful experiments with the $\mathrm{m} 3$ pi robots in the loop. The first experiment uses a hardware sheep robot, controlled by simulated herders, as shown in Figure 10. The second experiment uses two hardware dog robots herding a simulated sheep, shown in Figure 13. These are preliminary results, and for the final version of the paper, we anticipate additional experiments in which all agents in the system are m3pi robots.

\section{A. m3pi Sheep, Simulated Dogs}

Our first experiment uses a single $\mathrm{m} 3$ pi robot to represent the sheep, with $m=4$ simulated herders. The stills in Figure 10 illustrate the evolution of the system over time. Positions of the simulated herders are used to calculate the effective forces on the sheep, which are then transmitted to the m3pi. OptiTrack position data on the robot is read back into Matlab, which updates the herders. In the first frame of Figure 10, we have highlighted the starting positions of the simulated dog herders (blue squares) as well as the robotic sheep (red

\footnotetext{
${ }_{1}$ ww . naturalpoint. com/optitrack

${ }^{2}$ wWw. pololu.com

${ }^{3}$ www.digi.com/xbee
} 
circle). The goal region representing $B_{\ell}(g)$ is indicated by the green circle. Over the course of the experiment, we see the herders are able to successfully relocate the sheep to the desired goal region.

Figure 11 displays the time history of the m3pi sheep and simulated dogs over the course of the experiment. Here, the trajectories are noisier than those seen in the simulations. The additional noise comes from the unmodeled dynamics, including significant friction in the $\mathrm{m} 3$ pi robots and significant communication delays, that exist within the experimental system. This demonstrates an inherent robustness in our feedback controllers to tolerate uncertainty in our system.

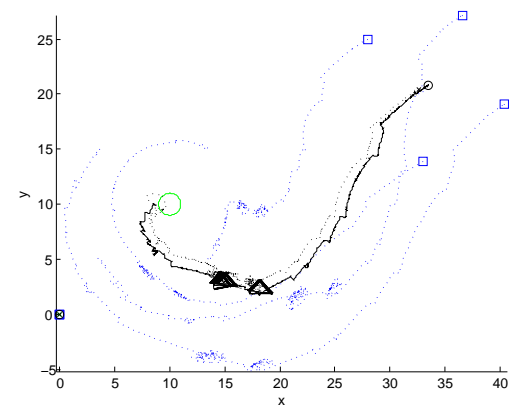

Fig. 11. Time history of the simulated robots (blue squares) and the m3pi sheep (black circle). Note the trajectories spiral towards the goal region.

We can also assess the performance by looking at the distance of the sheep's point-offset $p$ from the goal, as shown in Figure 12. From this figure, we see the distance decreases over time, indicating the sheep was successfully relocated to the goal region by the herders. Although there is chatter present, most noticeably between $t=50$ and $t=70$ seconds, it does not impact the overall performance of the controllers.

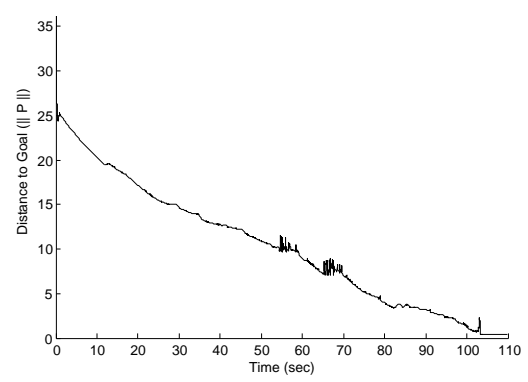

Fig. 12. Distance of the sheep's point offset $p$ to the goal.

\section{B. Simulated Sheep, Two m3pi Dogs}

Our second experiment uses a single simulated sheep, and $m=2 \mathrm{~m} 3$ pi robots as dogs. The stills in Figure 13 show the evolution of the experiment, with the herder robots successfully relocating the sheep to the desired goal region. Figure 14 shows the trajectories of the three agents over time. We can see that from the starting configuration, the herders (blue squares) drive the sheep (black circle) towards the goal region. The chatter near the goal region is due to discretization and communication delays, however, despite these effects the herders maintain the sheep near the goal.

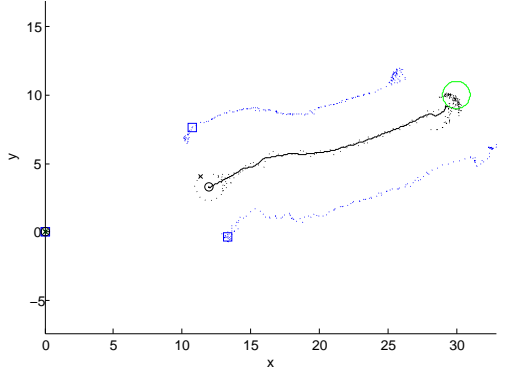

Fig. 14. Trajectories for $n=1$ simulated sheep (black circle) driven by $m=2 \mathrm{~m} 3$ pi herders (blue squares) towards the goal region.

As before, we can also examine the distance of the point offset to the goal, shown in Figure 15. Overall, we see the distance decreasing over time, illustrating that the sheep is relocated from its initial position to the goal region. Once the robot is close to the goal region, we notice some chatter in the system, due to the noisy actuation of the dog m3pi robots. Despite this noise, once the sheep is near the goal region, it never escapes.

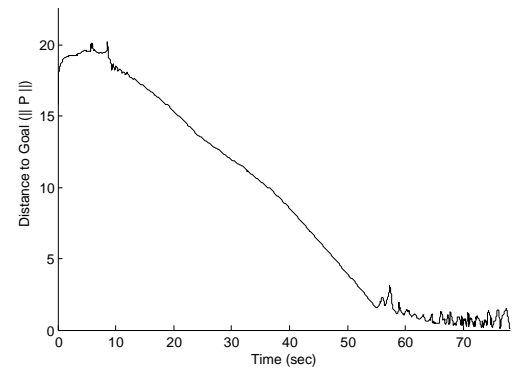

Fig. 15. Distance of the sheep's point offset $p$ to the goal.

\section{CONCLUSION}

We consider a scenario in which robotic dogs seek to control the location of a herd of non-cooperative sheep. The goal is for the herders to relocate the sheep to a region close to a goal point. Despite the highly nonlinear dynamics of the system, using the constraint that the dogs maintain some radius around the herd allows us to map these dynamics to unicycle-like dynamics, for which a simple feedback controller can be formulated. Unlike previous work in herding, this is done with a single continuous control law and does not rely on switching or heuristic behaviors. For a single sheep with multiple dogs, we are able to prove with a Lyapunovlike proof that the sheep converge asymptotically to the goal region. We also propose a control strategy for the general case of multiple sheep and multiple dogs. The simulations and hardware experiments demonstrate the performance of these control strategies.

In the future, we plan to analyze the convergence of the multi-sheep, multi-dog case. Other areas to investigate would be more complex problems for the herders to solve. For example, the herders may have to move the sheep along some trajectory or path in the environment, such as navigating through a maze. Another problem could be to avoid obstacles 


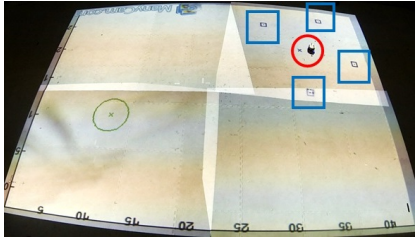

(a) $\mathrm{t}=4 \mathrm{sec}$

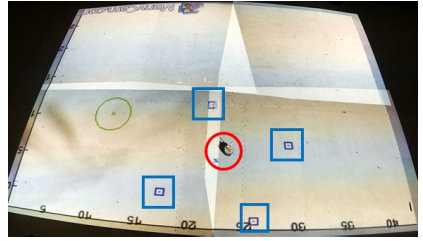

(b) $\mathrm{t}=45 \mathrm{sec}$

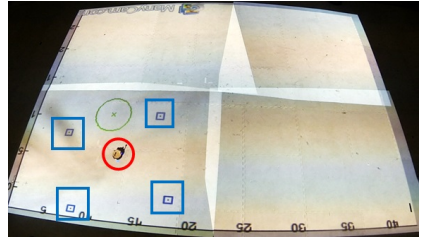

(c) $\mathrm{t}=80 \mathrm{sec}$

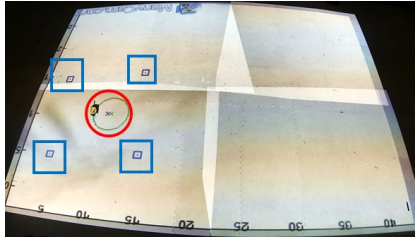

(d) $\mathrm{t}=110 \mathrm{sec}$

Fig. 10. Images from the experimental video, illustrating the herding of $n=1$ robot sheep (red circle) by $m=4$ simulated herders (blue squares).

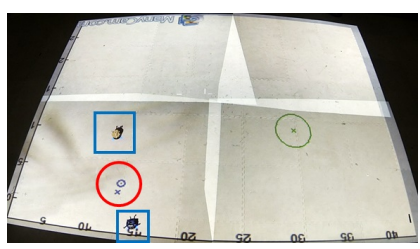

(a) $\mathrm{t}=7 \mathrm{sec}$

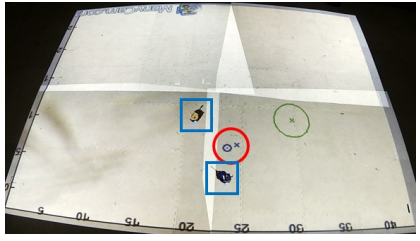

(b) $\mathrm{t}=45 \mathrm{sec}$

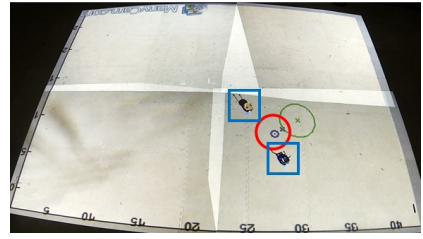

(c) $\mathrm{t}=55 \mathrm{sec}$

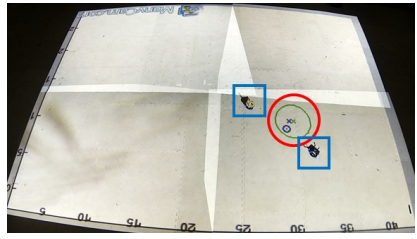

(d) $\mathrm{t}=70 \mathrm{sec}$

Fig. 13. Images from the experimental video, showing $m=2$ robotic dogs (blue squares) herding $n=1$ simulated sheep (red circle) to the goal.

or navigate around them. Beyond the relocation problems, there are also the herding problems of consolidating a sparse group of sheep, culling members from a group, or protecting the group from an external threat.

\section{ACKNOWLEDGMENTS}

This work was supported in part by ONR grant N0001412-1000, NSF grant CNS-1330036, and by a Clare Boothe Luce Fellowship. We are grateful for this support.

\section{REFERENCES}

[1] M. Lane. (2011, February) Helicopter cowboys of australia's outback. http://www.bbc.co.uk/news/world-asia-pacific12408888. [Online]. Available: http://www.bbc.co.uk/news/worldasia-pacific- 12408888

[2] A. Becker, C. Onyuksel, T. Bretl, and J. McLurkin, "Controlling many differential-drive robots with uniform control inputs," The International Journal of Robotics Research, 2014.

[3] R. Vaughan, "Experiments in automatic flock control," Ph.D. dissertation, University of Oxford, 1999.

[4] R. Vaughan, N. Sumpter, J. Henderson, A. Frost, and S. Cameron, "Experiments in automatic flock control," Robotics and Autonomous Systems, vol. 31, no. 1, pp. 109-117, 2000.

[5] J.-M. Lien, S. Rodriguez, J. Malric, and N. Amato, "Shepherding behaviors with multiple shepherds," in Robotics and Automation, 2005. ICRA 2005. Proceedings of the 2005 IEEE International Conference on, April 2005, pp. 3402-3407.

[6] S. A. Shedied, "Optimal control for a two-player dynamic pursuit evasion game: The herding problem," Ph.D. dissertation, Virginia Polytechnic Institute and State University, 2002, aAI3040288.

[7] Z. Lu, "Cooperative optimal path planning for herding problems," Master's thesis, Texas A\&M University, December 2006.

[8] M. Egerstedt and X. Hu, "Formation constrained multi-agent control," in Robotics and Automation, 2001. Proceedings 2001 ICRA. IEEE International Conference on, vol. 4, 2001, pp. 3961-3966 vol.4.

[9] G. Ferrari-Trecate, M. Egerstedt, A. Buffa, and M. Ji, "Laplacian sheep: A hybrid, stop-go policy for leader-based containment control," in Hybrid Systems: Computation and Control, ser. Lecture Notes in Computer Science, J. Hespanha and A. Tiwari, Eds. Springer Berlin Heidelberg, 2006, vol. 3927, pp. 212-226.

[10] C. M. Breder, "Equations descriptive of fish schools and other animal aggregations," Ecology, vol. 35, no. 3, pp. 361-370, Jul. 1954.

[11] V. Gazi and K. M. Passino, "Stability analysis of social foraging swarms," IEEE TRANS. ON SYSTEMS, MAN AND CYBERNETICS, vol. 34, no. 1, pp. 539-557, 2004.

[12] J. Toner and Y. Tu, "Flocks, herds, and schools: A quantitative theory of flocking," Phys. Rev. E, vol. 58, no. 4, p. 4828, 1998.
[13] C. W. Reynolds, "Flocks, herds and schools: A distributed behavioral model," SIGGRAPH Comput. Graph., vol. 21, no. 4, pp. 25-34, Aug. 1987. [Online]. Available: http://doi.acm.org/10.1145/37402.37406

[14] A. Howard, M. Matari, and G. Sukhatme, "Mobile sensor network deployment using potential fields: A distributed, scalable solution to the area coverage problem," in Distributed Autonomous Robotic Systems 5, H. Asama, T. Arai, T. Fukuda, and T. Hasegawa, Eds. Springer Japan, 2002, pp. 299-308.

[15] H. Tanner, A. Jadbabaie, and G. Pappas, "Stable flocking of mobile agents, part i: fixed topology," in Decision and Control, 2003. Proceedings. 42nd IEEE Conference on, vol. 2, Dec 2003, pp. 2010-2015 Vol.2.

[16] _ - "Flocking in fixed and switching networks," Automatic Control, IEEE Transactions on, vol. 52, no. 5, pp. 863-868, May 2007.

[17] R. Olfati-Saber, "Flocking for multi-agent dynamic systems: Algorithms and theory," IEEE Transactions on Automatic Control, vol. 51, pp. 401-420, 2006.

[18] D. DeVon and T. Bretl, "Kinematic and dynamic control of a wheeled mobile robot," in Intelligent Robots and Systems, 2007. IROS 2007. IEEE/RSJ International Conference on, Oct 2007, pp. 4065-4070.

[19] R. M. Murray, S. S. Sastry, and L. Zexiang, A Mathematical Introduction to Robotic Manipulation, 1st ed. Boca Raton, FL, USA: CRC Press, Inc., 1994.

[20] R. W. Brockett, Asymptotic Stability and Feedback Stabilization. Defense Technical Information Center, 1983.

[21] J. Cameron and W. Book, "Optimal path planning for the motion of a wheel," in Robotics and Automation, 1994. Proceedings., 1994 IEEE International Conference on, May 1994, pp. 1574-1580 vol.2.

[22] J. Laumond, S. Sekhavat, and F. Lamiraux, "Guidelines in nonholonomic motion planning for mobile robots," in Robot Motion Planning and Control, ser. Lecture Notes in Control and Information Sciences, J.-P. Laumond, Ed. Springer Berlin Heidelberg, 1998, vol. 229, pp. $1-53$.

[23] D. Chwa, "Sliding-mode tracking control of nonholonomic wheeled mobile robots in polar coordinates," Control Systems Technology, IEEE Transactions on, vol. 12, no. 4, pp. 637-644, July 2004.

[24] M. Aicardi, G. Casalino, A. Bicchi, and A. Balestrino, "Closed loop steering of unicycle like vehicles via lyapunov techniques," Robotics Automation Magazine, IEEE, vol. 2, no. 1, pp. 27-35, Mar 1995.

[25] N. Michael and V. Kumar, "Planning and control of ensembles of robots with non-holonomic constraints," The International Journal of Robotics Research, vol. 28, no. 8, pp. 962-975, 2009. [Online]. Available: http://ijr.sagepub.com/content/28/8/962.abstract

[26] H. Khalil, Nonlinear Systems. Prentice Hall PTR, 2002. 